\title{
A Deterministic Sensor Deployment Method for Target Coverage
}

\author{
Ye Jiang, ${ }^{1}$ Shuyan Xiao, ${ }^{2}$ Jian Liu, ${ }^{1}$ Bo Chen, ${ }^{1}$ Bangbang Zhang, ${ }^{1}$ Hongzhi Zhao, \\ and Zhaoneng Jiang $\mathbb{D}^{1}$ \\ ${ }^{1}$ Hefei University of Technology, Hefei 230009, China \\ ${ }^{2}$ Jiangsu University of Technology, Changzhou 213125, China
}

Correspondence should be addressed to Zhaoneng Jiang; jiangzhaoneng@hfut.edu.cn

Received 17 October 2017; Revised 25 January 2018; Accepted 19 February 2018; Published 26 April 2018

Academic Editor: Fanli Meng

Copyright ( $\odot 2018$ Ye Jiang et al. This is an open access article distributed under the Creative Commons Attribution License, which permits unrestricted use, distribution, and reproduction in any medium, provided the original work is properly cited.

\begin{abstract}
In order to monitor the gas leakage, the gas sensors are deployed conventionally in chemical industry park, with little considerations given to the gas characteristics and weather conditions, which give rise to the problems of coverage hole and coverage repetition. To solve the problems, this paper proposes a deterministic sensor deployment method with the gas diffusion models which takes into account wind speed and direction and then studies the influence of wind speed and direction on the monitoring error of gas sensors. Then, we research the deterministic deployment method of gas sensors in condition of the main wind speed and direction somewhere. Firstly, we use the CFD theory to simulate the gas diffusion situation so as to obtain the concentration value of the relevant points. Secondly, we put forward a new optimization criterion, namely, the more alarm concentration points covered by gas sensors, the coverage performance is better, and the deployment method is better. Accordingly, a new objection function is built. Thirdly, we obtain the weight values of the function using entropy estimation method. Finally, we deploy the gas sensors determinately using particle swarm optimization (PSO) algorithm. The simulation results show that the proposed method can improve the monitoring efficiency and the coverage performance of gas sensor network.
\end{abstract}

\section{Introduction}

In order to monitor the gas leakage, the gas sensors will be installed in the chemical industrial park according to some conventional industry standards, and the gas sensors act as alarm to provide some guidance for the supervisors when the leakage accidents happened. However, the health and safety executive (HSE) pointed out in 1993 and 2003 [1] that more than half gas leakage accidents happened in the chemical industrial parks cannot be detected on the grounds that the current deployment methods do not take the factors affecting gas diffusion into account, and the factors include the following: leakage source locations, the number of leakage sources, gas composition, weather conditions, design of pipeline, process conditions, building structure, isolation system, vacuum system, and monitoring frequency. Due to the influence of the factors on the monitoring efficiency, the deterministic deployment method should be considered.

The deployment of wireless sensor networks (WSN) can be mainly divided into two types according to the type of sensors, the application background, and the environment condition: deterministic deployment method and stochastic deployment method. We mainly adopt the deterministic deployment method in the cases where locations of sensors have a great impact on the operation of the WSN, such as deployment of sensor nodes on the pipe [2,3], deployment of image/video sensors indoors [4-6], deployment of underwater sensors [7], and deployment of seismic sensors for volcano monitoring [8]. However, we adopt the stochastic deployment method in some special occasions [9, 10], especially when the gas leakage happened in the chemical industrial parks. When the wind speed and direction influence the gas diffusion model, the sensor nodes deployed may be invalid, and the research of the deterministic deployment method is very important.

Aimed at the problem of hazard gas diffusion in the chemical industrial park, there are also many methods that study the deployment of gas sensors considering the gas diffusion model [11], including the heuristic analysis method [12], which is only designed for the serious leakage scenario, 
without considering the uncertainty of the leakage scenario. Besides, there is a method based on the theory of risk analysis that is proposed by the US International Standard Committee, wherein risk is the objective function to be minimized, and the disadvantage thereof is that the method can only get local optimal solution. And there is also a mixed integer linear programming by Legg and others according to the sensor deployment scheme of water pollution, which considers the impact of node failure on the network, but cannot consider the time series and is not in conformity with the actual situation, just like the assumption of node failure probability. The methods above are based on the simple diffusion model and with no consideration of environmental features such as wind speed and direction, so the node deployment is not accurate.

The main purpose of the WSN is to meet such demand of network performance as assuring integrity of the transmission data, reducing the delay time, reducing power consumption, prolonging the network lifetime, and increasing the coverage. Usually, we adopt the deterministic deployment method to meet these demands, and the optimization targets of the deterministic deployment mainly are coverage, connectivity, lifetime, and so on. All the deployment methods are desired to satisfy the demands with high efficiency, and the deployment methods designed by scholars can improve such performance of the WSN as better connectivity and longer lifetime [13-16]. Besides, there are some studies mainly about the load balance and fault tolerance $[17,18]$.

The coverage of WSN directly influences the possibility to monitor the target accurately and provide the highquality perceived service and also directly reflects the working performance of the WSN. Usually, the coverage ratio represents the performance of the coverage, and to maximize the coverage of monitoring targets is a main goal of the deployment of WSN. To solve the problem, there are many researches at home and abroad, which are mainly based on such perception model of the sensors such as Boolean disc model [19], directed model [20], and three dimensional sphere model [21], and there are also many researches based on such characteristics of monitoring targets as fixed monitoring points, mobile monitoring points, and monitoring areas that correspond to target coverage problems [22-26], barrier coverage problems [9, 27, 28], and area coverage problems $[29,30]$.

The aim of target coverage is to cover some discrete points whose locations are known, the classical Art Gallery Guard Problem [24] is aimed at solving the problem above, and in order to achieve the max coverage ratio, the paper [31] proposed the minimum cost based on integer linear programming, and the paper [22] provided a randomized algorithm to solve the coverage problem. And the coverage problem in the chemical industrial park is a critical problem we need to consider. The deterministic deployment has been little pursued in conventional deployment methods, and the coverage ratio is quite low. Aimed at this problem, we see the deployment problem as the Art Gallery Guard Problem; the location of the alarm gas concentration points in the chemical industrial park acts as the pictures in the art gallery, the sensors deployed act as the video cameras, and the locations of the cameras maximize the coverage ratio and achieve the balanced coverage.

The innovative points of this paper are listed as follows:

(1) We obtain the deterministic deployment method based on the gas diffusion model and research the influence of the wind speed and direction on the monitoring performance of the deployment method.

(2) We get the locations of the alarm gas concentration points simulated through the simulation of gas diffusion using the CFD theory.

(3) In order to solve the coverage hole problem, we propose a new optimization criterion, namely, the more alarm concentration points are covered by gas sensors and the more balanced the coverage is, the more optimal the deployment scheme is.

(4) We put forward a new objective function based on the new optimization criterion; meanwhile, we get the weight values of the function using the entropy estimation method.

(5) We provide a new deterministic deployment method based on the PSO algorithm and compare the new deterministic deployment method with the conventional deployment method and find that the performance of the method we proposed is better.

The next section of this paper is to first introduce the particle swarm optimization algorithm in Section 2. And we introduce the gas diffusion model considering the wind speed and direction in Section 3. In Section 4, we first put forward a new deterministic deployment method based on the gas diffusion model, then we research the influence of the wind speed and direction on the monitoring performance of the method proposed and conduct the simulated experiments and analysis. Aimed at the special wind speed and direction somewhere, we propose a deterministic deployment method to solve the coverage hole and unbalanced coverage in Section 5. In Section 6, we also compare the method we proposed and the conventional deployment methods to see the differences there between in terms of the performance of the coverage ratio and coverage balance. At last, we conclude the paper in Section 7.

\section{Particle Swarm Optimization Algorithm}

Particle swarm optimization algorithm (PSO) is developed by an American social psychologist James Kennedy and an electrical engineer Russell Beernaert [32, 33] in 1995; the basic idea is inspired by the results of the simulation of their early study and modeling of group behavior of many birds. PSO algorithm is a new evolutionary computation technique based on swarm intelligence, the optimization research is instructed by swarm intelligence produced by cooperation and competition among swarms in colony, and it has some features such as fast convergence speed, implementation simplicity, less parameter, and strong commonality. And now 
the PSO algorithm has attracted more attention and made great progress so as to be widely applicable in recent years.

The basic ideas of PSO are that each particle represents a feasible solution of the objective function, each particle has a fitness value determined by the objective function, and each particle has a speed within the scope of the solution. By changing speed constantly to traverse the feasible region, all the particles can search the best value in the current fitness value. In each iteration, every particle corresponds to a fitness value, also there is a global optimal fitness value, and the corresponding solutions of the global optimal fitness value are the global optimal positions of the sensors. Every iteration updates constantly based on their locations and global optimal location until finding the required optimal solution, then we can end the iteration.

We assume that the particles search optimal solution in a $D$ dimensional space, and the specific process of PSO algorithm is as follows:

(1) Determine the objective function $f\left(x_{1}, \ldots, x_{D}\right)$.

(2) Initialize the positions of $M$ particles $P_{1}=\left(x_{1}, \ldots\right.$, $\left.x_{D}\right), P_{2}=\left(x_{21}, \ldots, x_{2 D}\right), \ldots, P_{M}=\left(x_{M 1}, \ldots, x_{M D}\right)$.

(3) Initialize each dimension velocity of every particle $v_{P 1}=\left(v_{1}, \ldots, v_{D}\right), v_{P 2}=\left(v_{21}, \ldots, v_{2 D}\right), \ldots, v_{P M}=$ $\left(v_{M 1}, \ldots, v_{M D}\right)$.

(4) Calculate the fitness value of each particle $P_{1}, P_{2}, \ldots$, $P_{M}$.

(5) Compare each particle's own fitness and get the global optimal value gbest.

(6) Update the speed according to the velocity update formula $v_{i d}^{k+1}=v_{i d}^{k}+c_{1} \xi\left(p_{i d}^{k}-x_{i d}^{k}\right)+c_{2} \eta\left(g_{i d}^{k}-x_{i d}^{k}\right)$.

(7) Update the particle's position according to the location updating formula $x_{i d}^{k+1}=x_{i d}^{k}+r v_{i d}^{k+1}$.

As for steps 6 and 7, we illustrate as follows:

(1) $i$ means the $i$ th particle, $d$ means the $d$ th element of the particle, and $k$ means the $k$ th iteration.

(2) $c_{1}$ means the cognition of the particle itself, which is generally set to be 2 .

(3) $c_{2}$ means the overall recognition of the particle swarm, which is usually set to be 2 .

(4) $\xi$ and $\eta$ are random numbers in the range $[0,1]$.

(5) $r$ means a coefficient added in front of the speed when updating the position, namely, a constraint factor, which is generally set to be 1 .

(6) $v_{i d}$ is the particle's velocity, in the range $\left[v_{\min }, v_{\max }\right]$, when $v_{i d}>v_{\max }, v_{i d}=v_{\max }$, and when $v_{i d}<v_{\min }$, $v_{i d}=v_{\min }$.

(7) As for the velocity update formula $v_{i d}^{k+1}=v_{i d}^{k}+$ $c_{1} \xi\left(p_{i d}^{k}-x_{i d}^{k}\right)+c_{2} \eta\left(g_{i d}^{k}-x_{i d}^{k}\right)$, we usually use the improved formula which is $v_{i d}^{k+1}=w_{k} v_{i d}^{k}+c_{1} \xi\left(p_{i d}^{k}\right.$ $\left.-x_{i d}^{k}\right)+c_{2} \eta\left(g_{i d}^{k}-x_{i d}^{k}\right)$, and $w_{k}$ is inertia weighting factor [34]. At the beginning of the iteration, the inertia weighting factor is large, which has a good convergence speed for global search. With the increase of the number of iterations, the inertia weighting factor decreases, which has a good convergence speed for local search. The paper [35] proposed a PSO algorithm with linear decreasing inertia weight.

$w_{k}=0.9-k / \max$ gen $\times 0.5$, where max gen is the number of max iterations.

The paper [36] also proposed inertia weight nonlinear dynamic adjustment method based on fuzzy system.

\section{The Establishment of Gas Diffusion Model considering Meteorological Conditions}

The gas diffusion model can be set up based on certain diffusion equations and some conditions, but due to such weather conditions having impact on the gas diffusion model as wind speed and direction, the previous diffusion model is not applicable, which may have the influence on the gas sensor deployment. The deployment of sensors cannot effectively monitor a gas leak.

The papers $[37,38]$ researched the gas diffusion mechanism sufficiently. The diffusion model without considering meteorological conditions is as follows:

$$
\begin{aligned}
c(\vec{r}, t)= & \frac{\mu}{4 \times \pi \times \kappa\left|\vec{r}-\vec{r}_{0}\right|} \operatorname{erf} c\left(\frac{\left|\vec{r}-\vec{r}_{0}\right|}{2 \sqrt{\kappa\left(t-t_{0}\right)}}\right) \\
& +\frac{\mu}{4 \times \pi \times \kappa\left|\vec{r}-\vec{r}_{1}\right|} \operatorname{erf} c\left(\frac{\left|\vec{r}-\vec{r}_{1}\right|}{2 \sqrt{\kappa\left(t-t_{0}\right)}}\right) .
\end{aligned}
$$

$c(\vec{r}, t)$ is the concentration of gas source in space location of $\vec{r}=[x, y, z]^{T}$ at $t$ time, $\vec{r}_{0}=\left[x_{0}, t_{0}, z_{0}\right]^{T}$ and $\vec{r}_{1}=$ $\left[x_{0}, y_{0},-z_{0}\right]^{T}$ are the positions of the gas source and their image positions relative to an interface, respectively, $k$ is the rate of diffusion of the gas source, the unit of the diffusion rate is $\mathrm{m}^{2} / \mathrm{s}$, the diffusion rate will vary with the change of environmental temperature, $\mu$ is the quality release rate of the gas source and the unit thereof is $\mathrm{kg} / \mathrm{s}$, and $t_{0}$ is the start time of gas source.

Taking wind speed and direction into consideration, wind speed vector is changed to be parallel to axis $x$ by coordinate transformation, and $v_{x}$ denotes wind velocity; we assume that the gas source and the sensor nodes are in the same plane, namely, $z=z_{0}=0$; when the gas reached the steady state, concentration of gas diffusion can be simplified as follows:

$$
c(\vec{r}, t)=\frac{\mu \times \exp \left(-V_{x} / 2 \times k\left(d-\left(x-x_{0}\right)\right)\right)}{2 \pi k d} .
$$

$v_{x}$ is wind velocity paralleled to axis $x$ after coordinate transformation, $x$ and $x_{0}$ are abscissas of the sensor and the leakage source after coordinate transformation, $\left(x-x_{0}\right)$ is the 
difference between the leakage source and sensor nodes after the coordinate transformation, $d$ is the distance between gas source and sensor nodes, and the coordinate transformations are as follows:

$$
\begin{aligned}
& x=x^{\prime} \times \cos (\theta(t))+y^{\prime} \times \sin (\theta(t)), \\
& x_{0}=x_{0}^{\prime} \times \cos (\theta(t))+y_{0}^{\prime} \times \sin (\theta(t)), \\
& d=\left\|r^{\prime}-r_{0}^{\prime}\right\|=\sqrt{\left(x^{\prime}-x_{0}^{\prime}\right)^{2}+\left(y^{\prime}-y_{0}^{\prime}\right)^{2}} .
\end{aligned}
$$

$\left(x^{\prime}, y^{\prime}\right)$ and $\left(x_{0}^{\prime}, y_{0}^{\prime}\right)$ are the position coordinates of the sensor nodes and the leakage source, respectively.

Prediction model is as follows:

$$
c_{1}(\vec{r}, t)=\frac{\mu \times \exp \left(-V_{x} / 2 \times k\left(d-\left(x^{\prime}-x_{0}^{\prime}\right)\right)\right)}{2 \pi k d} .
$$

$v_{x}$ is the wind velocity parallel to axis $x$ after coordinate transformation.

Measurement model is as follows:

$$
c_{2}(\vec{r}, t)=\frac{\mu \times \exp \left(-V_{x} / 2 \times k\left(d-\left(\left(x^{\prime} \times \cos (\theta(t))+y^{\prime} \times \sin (\theta(t))\right)-\left(x_{0}^{\prime} \times \cos (\theta(t))+y_{0}^{\prime} \times \sin (\theta(t))\right)\right)\right)\right.}{2 \pi k d} .
$$

$\theta(t)$ is the wind direction which changes over time anticlockwise.

\section{The Simulation Experiments and Analysis of the Influence of the Meteorological Conditions on the Error Rate in the Deterministic Optimization Deployment Method of Gas Sensors}

4.1. The Simulation Scenario and Parameter Setting of the Deterministic Optimization Deployment Method. We simulate a propane gas leakage in a chemical industrial park, wherein the simulation area is $100 \mathrm{~m} \times 100 \mathrm{~m}$. There is only a leakage source, the number of sensors is changed according to the purpose of the experiment, which is in the range of [20, 39], diffusion time is set from $10 \mathrm{~s}$ to $50 \mathrm{~s}$, and the number of particles is set from 20 to 50 according to the purpose of the experiment. The wind velocity in the prediction model is set to be $2 \mathrm{~m} / \mathrm{s}$, and the wind is blown to the $x$ negative axis. The wind velocity of the measurement model is set to be $v_{x}=2$ $\mathrm{m} / \mathrm{s}+a \times t ; a$ varies with the purpose of the experiment which is within the range of $[0.2,1] \mathrm{m} / \mathrm{s}^{2}$. The wind direction is set to be $\theta(t)=(\pi / n) \times t$, the wind is blown to $x$ negative axis and varies anticlockwise, $n$ varies with the purpose of the experiment which is within the range of $[18,90]$, and both of the two parameters above vary over time. Gas source diffusion rate is $k=50 \mathrm{~m}^{2} / \mathrm{s}$, and the leaking source has an intensity of $20 \mathrm{~kg} / \mathrm{s}$, namely, $\mu=20 \mathrm{~kg} / \mathrm{s}$; the value of inertia weight factor $w$ is 0.729 , and the constant $c_{1}$ and $c_{2}$ are both set to be 1.496 . And the number of iterations in every experiment is 500. All of the simulation experiments are completed by MATLAB software.

(1) Establish the objective function: Based on PSO optimization algorithm, the most important thing is to establish the objective function. This paper makes the average error of the prediction model and the measurement model as the objective function. There are $N$ sensor nodes, the deployed locations of the nodes are used as the solution vector, and the average concentration error rate of the $N$ sensors during the diffusion time is the objective function, which is made to be a minimum. The expression of prediction model is $C_{1}$, and the expression of measurement model is $C_{2}$.

The objective function is as follows:

$$
\text { Minimize } \quad J=\frac{\sum_{i=1}^{N} \sum_{j=1}^{M} \sum_{t=1}^{\mathrm{time}}\left|C_{1}(i, j, t)-C_{2}(i, j, t)\right|}{N \times c_{2}(i, j) \times \text { time }},
$$

where $N$ is the number of gas sensors, $M$ is the number of the gas leakage sources, and time is denotes the gas diffusion time.

(2) $M$ particles are initialized to $D$-dimension matrixes randomly, which include the initial coordinates, the current coordinates, and the speed of each coordinate, as well as the individual fitness value and global best fitness value. The form is as follows:

$$
\begin{aligned}
P_{1}= & {\left[\left(x_{1}, y_{1}\right), \ldots,\left(x_{N}, y_{N}\right),\left(x_{1}^{\prime}, y_{1}^{\prime}\right), \ldots,\right.} \\
& \left.\left(x_{N}^{\prime}, y_{N}^{\prime}\right),\left(v_{1}, \ldots, v_{N}\right), \text { Pbest, gbest }\right],
\end{aligned}
$$

where $\left(x_{1}, y_{1}\right), \ldots,\left(x_{N}, y_{N}\right)$ is the initial coordinates, $\left(x_{1}^{\prime}, y_{1}^{\prime}\right), \ldots,\left(x_{N}^{\prime}, y_{N}^{\prime}\right)$ is the current coordinates, $\left(v_{1}\right.$, $\left.\ldots, v_{N}\right)$ is the speed of each coordinate, Pbest is an individual fitness value, and gbest is the global best fitness value.

(3) Calculate the fitness value of each particle $\left[\text { Pbest }_{1}, \text { Pbest }_{2}, \ldots, \text { Pbest }_{M}\right]^{\prime}$.

(4) Compare each particle's own fitness value and get the global best fitness value gbest.

(5) Update the velocity according to the velocity update formula: $v_{i d}^{k+1}=w_{k} \times v_{i d}^{k}+c_{1} \xi\left(p_{i d}^{k}-x_{i d}^{k}\right)+c_{2} \eta$ $\left(g_{i d}^{k}-x_{i d}^{k}\right)$. 
(6) Update the particle's position according to the location update formula: $x_{i d}^{k+1}=x_{i d}^{k}+r v_{i d}^{k+1}$.

(7) Keep iterating until reaching the global optimal value and obtaining the corresponding coordinates.

4.2. The Influence of the Wind Speed on Error Rate of Concentration in Deterministic Deployment Method. Under the condition that the number of the particles and the sensors are invariable and under different acceleration, first, we investigate the variation between the error rate of concentration in the deterministic deployment method and the time. The number of sensors is 20 , the number of particles is 20 , the diffusion time is in range of [10 s, $50 \mathrm{~s}]$, the wind direction is constant, the wind speed varies according to the equation $v_{x}=2 \mathrm{~m} / \mathrm{s}+a \times t$, where $a$ is the acceleration in the range of $\left[0 \mathrm{~m} / \mathrm{s}^{2}, 1 \mathrm{~m} /{ }^{2}\right]$, and the variation curve is shown in Figure 1.

As shown in Figure 1, we can know that the error rate of concentration increases with the increase of the acceleration. The maximum error rate is below $10 \%$. According to the alarm time, we can choose the corresponding deployment method under the different wind speeds.

Then we research the variation between the number of particles and the error rate of concentration under different wind speeds when there are 20 sensors and the diffusion time is $30 \mathrm{~s}$; meanwhile, we research the variation between the number of sensors and the error rate of concentration under different wind speeds when there are 20 particles and the diffusion time is $30 \mathrm{~s}$. The variations are shown in Figure 2.

As shown in Figure 2(a), we can know that the error rate of concentration increases with the increase of the acceleration. The maximum error rate is below 10\%. And the error rate of concentration increases with the increase of the number of sensors. Therefore, we can choose the corresponding deployment method according to the required error rate of concentration.

As shown in Figure 2(b), we can know that the error rate of concentration increases with the increase of the acceleration. The maximum error rate is below $10 \%$. And the error rate of concentration decreases with the increase of the number of particles. Therefore, we can set the corresponding parameters of the algorithm according to the required error rate of concentration.

4.3. The Influence of the Wind Direction on the Error Rate of Concentration in Deterministic Deployment Method. Under the condition that the number of the particles and the sensors are invariable and under different wind directions, first, we investigate the variation between the error rate of concentration in the deterministic deployment method and the time. The number of sensors is 20 , the number of particles is 20 , the diffusion time is within the range of $[10 \mathrm{~s}, 50 \mathrm{~s}]$, the wind direction varies according to the equation $\theta(t)=(\pi / n) \times t, n$ varies within the range of $[18,90]$, and the variation of the error rate is shown in Figure 3.

As shown in Figure 3, we can know that the error rate of concentration increases with the increase of the wind direction. The maximum error rate is below 6\%. According to

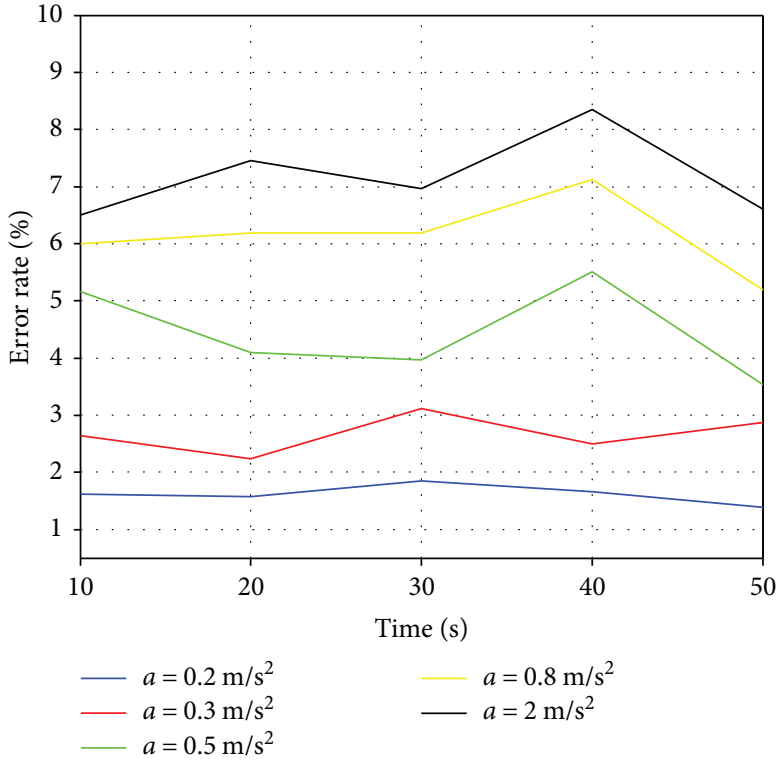

FIgURE 1: The variation curve between the error rate of concentration and time under different acceleration.

the alarm time, we can choose the corresponding deployment method under the different wind directions.

Then we research the variation between the number of sensors and the error rate of concentration under different wind directions when there are 20 particles and the diffusion time is $30 \mathrm{~s}$; meanwhile, we research the variation between the number of particles and the error rate of concentration under different wind directions when there are 20 sensors and the diffusion time is $30 \mathrm{~s}$. The variations are shown in Figure 4.

As shown in Figure 4(a), we can know that the error rate of concentration increases with the increase of the wind direction. The maximum error rate is below 6\%. And the error rate of concentration increases with the increase of the number of sensors. Therefore, we can choose the corresponding deployment method according to the required error rate of concentration.

As shown in Figure 4(b), we can know that the error rate of concentration increases with the increase of the wind direction. The maximum error rate is below 5\%. And the error rate of concentration decreases with the increase of the number of particles. Therefore, we can set the corresponding parameter of the algorithm according to the required error rate of concentration.

\section{The Deterministic Optimization Deployment Method of Gas Sensors Based on the Coverage Performance}

5.1. The Optimization Criterion and the Establishment of Objective Function. Due to the wind speed and direction, the leakage velocity, and the diffusion time factors, the hazardous gas diffusion models are different. Therefore, the gas concentrations in the same site are different, and the alarm 


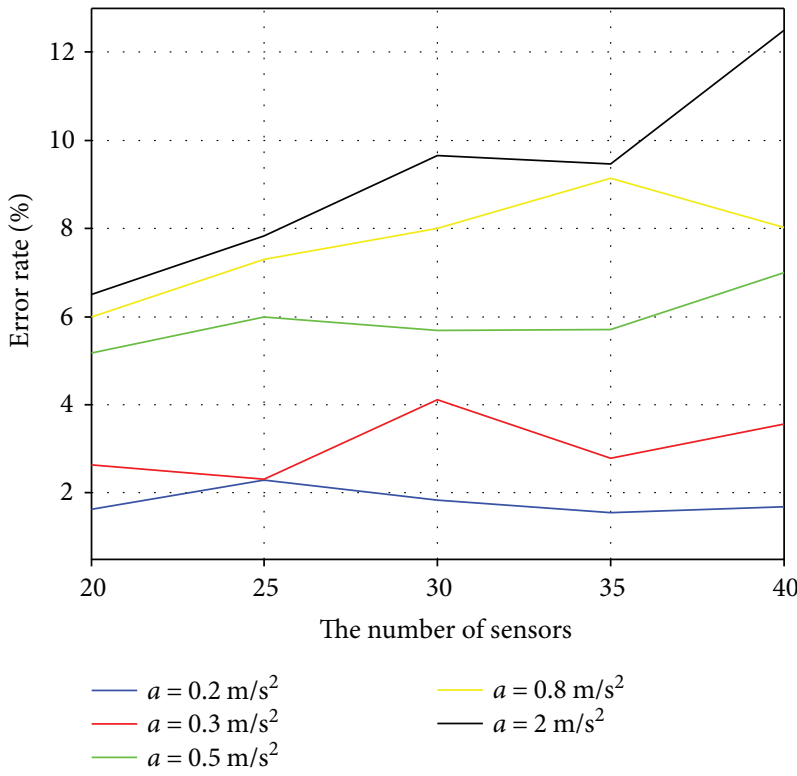

(a)

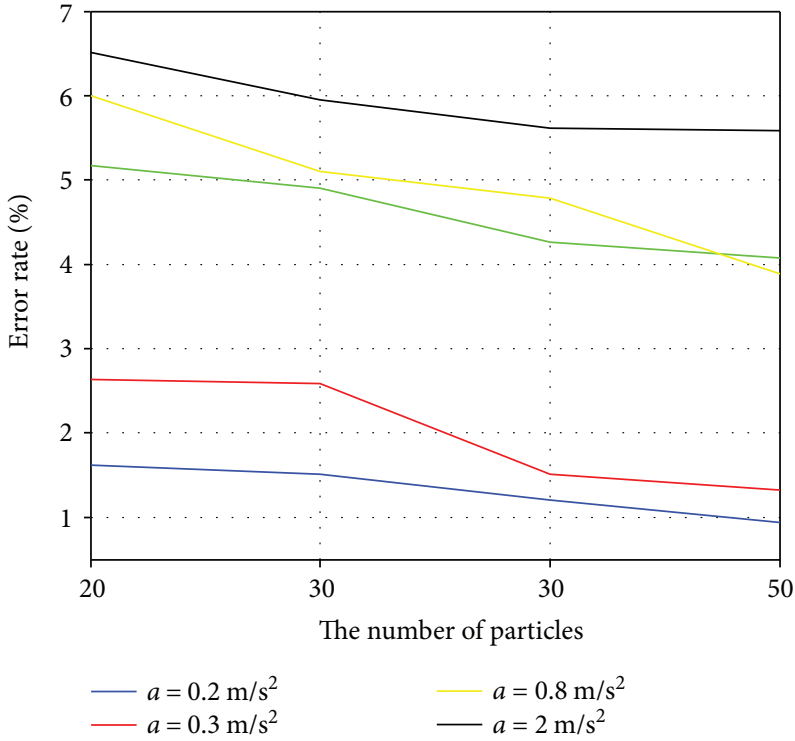

(b)

Figure 2: (a) The variation curve between the number of particles and the error rate of concentration under different wind speeds; (b) the variation curve between the number of particles and the error rate of concentration under different wind speeds.

concentration should be perceived by the gas sensors. The optimization criterion in this paper is as follows: (1) The more the number of the sensors monitored alarm concentration point, the better the deployment scheme. (2) The more balanced the coverage, the better the deployment scheme.

Based on the content above, there are $M$ alarm concentration points in the chemical industrial park expressed by $e_{i}, i \in(1,2, \ldots, M)$, the position coordinates of points are $\left[\left(x_{1}, y_{1}\right),\left(x_{2}, y_{2}\right), \ldots,\left(x_{M}, y_{M}\right)\right]$, respectively. And the number of the gas sensors is $N$ expressed by $s_{j}, j \in(1,2, \ldots, N)$. The perception radius of the sensors is $r$, and the position coordinates of the sensors are $\left[\left(x_{s 1}, y_{s 1}\right),\left(x_{s 2}, y_{s 2}\right), \ldots\right.$, $\left.\left(x_{s N}, y_{s N}\right)\right]$, respectively.

The alarm concentration point is covered by the gas sensor if the distance between the gas sensor and the point is less than $r$, namely, $d\left(e_{i}, s_{j}\right)<r$, wherein $d\left(e_{i}, s_{j}\right)$ denotes the distance between the $i$ th point and $j$ th sensors. Conversely, if the distance between the alarm concentration point and every gas sensors is more than $r$, the alarm concentration point is not covered by any gas sensors, and the deployment method is optimal if the number of the points is minimal.

The balanced coverage of gas sensors needs to meet the following condition: minimize $\left(\sum_{j=1}^{N} \sqrt{\left(c\left(s_{j}\right)-E(s)\right)^{2}} / N\right)$, wherein $c\left(s_{j}\right)$ denotes the number of the alarm concentration points covered by the $j$ th gas sensor, and $E(s)$ denotes the average value of the number of the alarm concentration points covered by all the gas sensors.

According to the content above, we can obtain the objective function as follows:

$$
\begin{aligned}
\text { Minimize } \quad F & =\alpha \times \frac{\sum_{j=1}^{N} \sqrt{\left(c\left(s_{j}\right)-E(s)\right)^{2}}}{N}+\beta \times \frac{\sum_{i=1}^{M} I\left(d\left(e_{i}, s_{1}\right)>\cap d\left(e_{i}, s_{2}\right)>r, \ldots, \cap d\left(e_{i}, s_{N}\right)>r\right)}{M} . \\
\text { Let } A & =\frac{\sum_{j=1}^{N} \sqrt{\left(c\left(s_{j}\right)-E(s)\right)^{2}}}{N}, \\
B & =\frac{\sum_{i=1}^{M} I\left(d\left(e_{i}, s_{1}\right)>r \cap\left(d\left(e_{i}, s_{2}\right)>r, \ldots, \cap d\left(e_{i}, s_{N}\right)>r\right)\right.}{M},
\end{aligned}
$$

where $A$ denotes the coverage balance degree, $B$ denotes the degree of coverage, and $\alpha$ and $\beta$ denote the weight values of $A$ and $B$ in the objective function. $I\left(d\left(e_{i}, s_{1}\right)>r \cap d\left(e_{i}, s_{2}\right)>\right.$ $\left.r, \ldots, \cap d\left(e_{i}, s_{N}\right)>r\right)=1$ denote the alarm concentration point, $e_{i}$ is not covered by any gas sensors, and the value is as follows: 


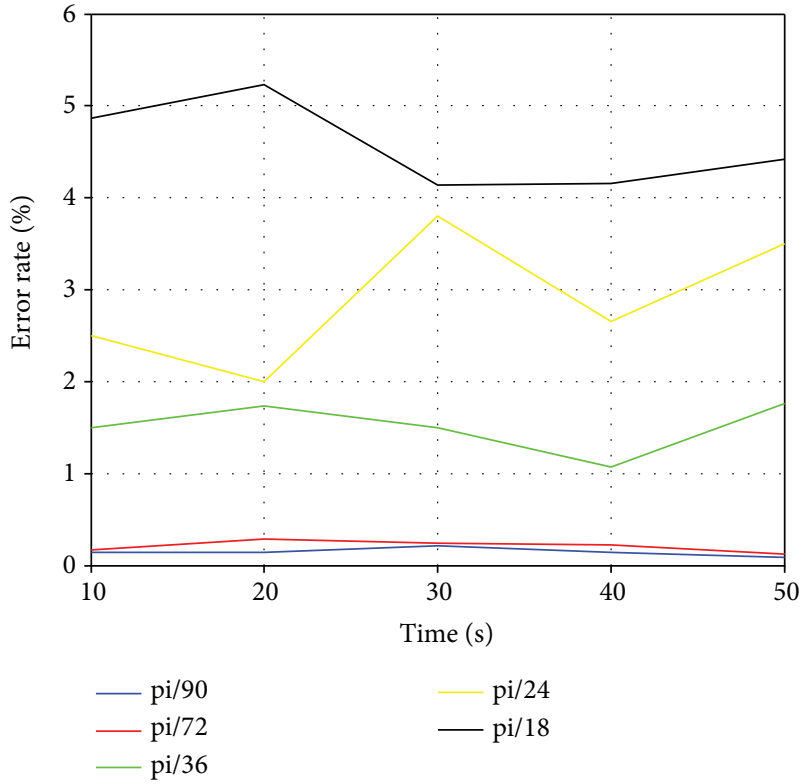

FIGURE 3: Diagram of error rate of concentration under different directions.

$$
\begin{aligned}
& I\left(d\left(e_{i}, s_{i}\right)>r \cap d\left(e_{i}, s_{2}\right)>r, \ldots, \cap d\left(e_{i}, s_{N}\right)>r\right) \\
& \quad= \begin{cases}1 & d\left(e_{i}, s_{1}\right)>r \cap d\left(e_{i}, s_{2}\right)>r, \ldots, \cap d\left(e_{i}, s_{N}\right)>r=1, \\
0 & d\left(e_{i}, s_{1}\right)>r \cap d\left(e_{i}, s_{2}\right)>r, \ldots, \cap d\left(e_{i}, s_{N}\right)>r=0,\end{cases}
\end{aligned}
$$

where $N$ denotes the number of gas sensors, and $M$ denotes the number of alarm concentration points.

\subsection{Determining the Weight Values of the Objective Function}

5.2.1. Simulation of Gas Leakage Scenario. We use the theory of computational fluid dynamics (CFD) to simulate the diffused propane in a park. The simulation zone size is
$50 \mathrm{~m} \times 50 \mathrm{~m} \times 10 \mathrm{~m}$, and the leaking source has an intensity of $14.28 \mathrm{~kg} / \mathrm{s}$ with the height of $2 \mathrm{~m}$, namely, $\mathrm{H}=2 \mathrm{~m}$, the wind speed is $1.5 \mathrm{~m} / \mathrm{s}$, when the gas diffusion time is $30 \mathrm{~s}$, and the gas diffusion condition is shown in Figure 5.

We set $z=0.6 \mathrm{~m}$ as the observation surface in the simulation and select lots of points on the surface to get the corresponding concentrations, as the measured gas concentration is the molar concentration having a unit of $\mathrm{kmol} /$ $\mathrm{m}^{3}$. According to the Chinese GB50493-2009, when the lower explosive limit (LEL) of propane gas is $2.1 \%$ (volume ratio mixed with air) the upper explosive limit (UEL) of propane gas is $9.5 \%$, we can obtain the alarm concentration according to the formula $V=C \times 22.4 \mathrm{~L} / \mathrm{mol}$, where $C$ is the measured gas concentration. Let $2.1 \%=C_{\min } \times 22.4 \mathrm{~L} / \mathrm{mol}$, $9.5 \%=C_{\max } \times 22.4 \mathrm{~L} / \mathrm{mol}, C_{\min }=9.375 \times 10^{-4} \mathrm{kmol} / \mathrm{m}^{3}$, and $C_{\max }=4.24 \times 10^{-3} \mathrm{kmol} / \mathrm{m}^{3}$; after calculation, the number of alarm concentration points is 39 , and the coordinates and the concentration are shown in Table 1.

5.2.2. Determining the Weight Values of the Objective Function Based on Entropy Estimation Method. We adopt the entropy estimation method [39] to calculate the weight values $\alpha$ and $\beta$ of the objective function, and the specific steps are as follows:

(1) Calculate the value of $A$ and $B$ under the condition that the numbers of sensors are 5, 8, 11, and 13 using the PSO algorithm.

The perceived radius of the gas sensors is $5 \mathrm{~m}$, namely, $r=5 \mathrm{~m}$. The number of alarm concentration points is 39 in Section 5.1, namely, $M=39$. The number of particles is 20 in PSO algorithm, and the number of iterations is 5000 . The global optimal value of the algorithm is obtained by repeating the experiment for 30 times. The steps are as follows.

(i) Establish the objective function: The objective function is as follows:

$$
\text { minimize } \quad F=\frac{\sum_{j=1}^{N}\left(c\left(s_{j}\right)-E(s)\right)}{N}+\frac{\sum_{i=1}^{M} I\left(d\left(e_{i}, s_{1}\right)>r \cap d\left(e_{i}, s_{2}\right)>r, \ldots, \cap d\left(e_{i}, s_{N}\right)>r\right)}{M} .
$$

The objective function is expressed as the coverage balance degree and the coverage ability of each gas sensor.

(ii) The 20 particles are randomly initialized to $D$ dimensional matrixes which include initial parameters as follows: $P_{1}=\left[\left(x_{1}, y_{1}\right), \ldots,\left(x_{N}, y_{N}\right)\right.$, $\left(x_{1}^{\prime}, y_{1}^{\prime}\right), \ldots,\left(x_{N}^{\prime}, y_{N}^{\prime}\right),\left(v_{1}, \ldots, v_{N}\right)$, Pbest, gbest $]$, where $\left(x_{1}, \mathrm{y}_{1}\right), \ldots,\left(x_{N}, y_{N}\right)$ are the initial coordinates, $\left(x_{1}^{\prime}, y_{1}^{\prime}\right), \ldots,\left(x_{N}^{\prime}, y_{N}^{\prime}\right)$ denote the current coordinates, $\left(v_{1}, \ldots, v_{N}\right)$ are the speeds of each coordinate, Pbest is the individual fitness value, and gbest is the global fitness value. $N$ is the number of the gas sensors. Because $z=0.6 \mathrm{~m}$, the $x$ and $y$ coordinates should be enough.

Please refer to Section 4.1 for the remaining steps.

After calculation, the values of $A$ and $B$ are shown in Table 2 under the different number of gas sensors. 
(2) Standardize the data: Standardize the values of $A$ and $B$ in step 1 using the following:

$$
X_{i j}^{\prime}=\frac{X_{i j}}{\sum_{j=1}^{2} X_{i j}},
$$

where $i$ denotes the case in which the number of gas sensors is different. It represents $A$ when $j=1$, and it represents $B$ when $j=2$. The standardized values are shown in Table 3.

(3) Calculate the ratio of $A$ and $B$ according to the number of gas sensors.

Calculate the ratio of $A$ and $B$ using the following:

$$
Y_{i j}=\frac{X_{i j}^{\prime}}{\sum_{i=1}^{m} X_{i j}^{\prime}},
$$

where $m$ denotes the case in which the number of gas sensors is different, which is 5 in this paper.

(4) Calculate the information entropy of each section.

Calculate the information entropy of the $j$ th section using the following:

$$
e_{j}=-k \sum_{i=1}^{m} Y_{i j} \times \ln Y_{i j} .
$$

(5) Calculate the redundancy of the information entropy.

Calculate the redundancy of the information entropy using the following:

$$
d_{j}=1-e_{j} .
$$

(6) Calculate the weight value of each section.

Calculate the weight value of the $j$ th section using the following:

$$
W_{i}=\frac{d_{j}}{\sum_{j=1}^{2} d j} .
$$

After these steps above, we can obtain the weight values of $A$ and $B$ in objective function, which are shown in Table 4.

5.3. Deterministic Optimization Deployment Method. The steps of the optimization deployment method are follows:

(1) Establish the experiment objective function: According to the weight values obtained in Section 5.2, the objective function based on the PSO algorithm is as follows:

Minimize $\quad F=0.4886 \times \frac{\sum_{j=1}^{N} \sqrt{\left(c\left(s_{j}\right)-E(s)\right)^{2}}}{N}+0.5114 \times \frac{\sum_{i=1}^{M} I\left(d\left(e_{i}, s_{1}\right)>r \cap d\left(e_{i}, s_{2}\right)>r, \ldots, \cap d\left(e_{i}, s_{N}\right)>r\right)}{M}$.

The objective function is expressed as the coverage balance degree and the coverage ability of each gas sensor.

(2) The 20 particles are randomly initialized to $D$-dimensional matrixes which include initial parameters as follows: $P_{1}=\left[\left(x_{1}, y_{1}\right), \ldots,\left(x_{N}, y_{N}\right),\left(x_{1}^{\prime}, y_{1}^{\prime}\right), \ldots,\left(x_{N}^{\prime}\right.\right.$, $\left.y_{N}^{\prime}\right),\left(v_{1}, \ldots, v_{N}\right)$, Pbest, gbest $]$, where $\left(x_{1}, y_{1}\right), \ldots$, $\left(x_{N}, y_{N}\right)$ are the initial coordinates, $\left(x_{1}^{\prime}, y_{1}^{\prime}\right), \ldots,\left(x_{N}^{\prime}\right.$, $\left.y_{N}^{\prime}\right)$ denote the current coordinates, $\left(v_{1}, \ldots, v_{N}\right)$ are the speeds of each coordinate, Pbest is the individual fitness value, and gbest is the global fitness value. $N$ is the number of the gas sensors.

Please refer to Section 4.1 for the remaining steps.

\section{The Simulation and Analysis}

6.1. The Simulation Scene and Parameter Setting. We simulate the propane gas leakage. The simulation zone is
$50 \mathrm{~m} \times 50 \mathrm{~m} \times 10 \mathrm{~m}$, the leaking source has an intensity of $14.28 \mathrm{~kg} / \mathrm{s}$, the wind speed is $1.5 \mathrm{~m} / \mathrm{s}$, the wind is blown to the $x$ positive axis, and the gas diffusion time is $30 \mathrm{~s}$. The number of particles is 20 in the PSO algorithm, the number of sensors is 8 , and the number of iterations is 5000 in every experiment. Because $z=0.6 \mathrm{~m}$, the $x$ and $y$ coordinates should be enough.

6.2. The Comparison of Different Deployment Method. The optimal deployment method proposed in this paper based on the PSO algorithm is shown in Figure 6(a), and according to the Chinese GB50493-2009 and the Application Data Sheet ADS-001 Gas Sensor Placement Guidelines, multiple gas sensor monitoring networks are deployed usually in the form of the rectangle or sector, which are shown in Figures 6(b) and 6(c).

As shown in Figure 6(a), the red spots represent the alarm concentration points, and the blue spots represent the gas sensors deployed in the park. And the position coordinates of the eight gas sensors are shown in Table 5. 


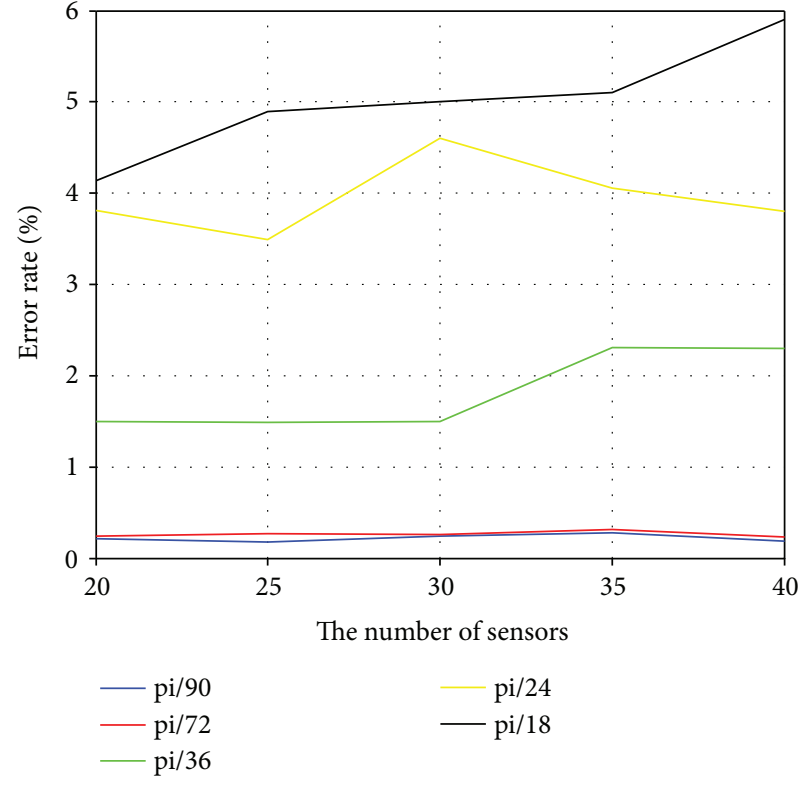

(a)

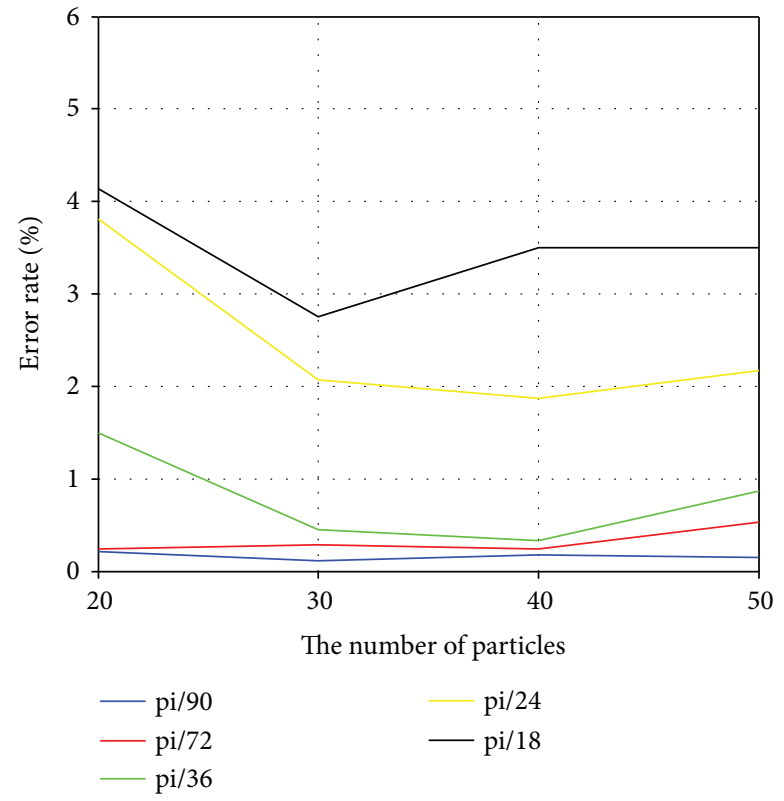

(b)

FIGURE 4: (a) The variation curve between the number of sensors and the monitored error rate under different wind directions; (b) the variation curve between the number of sensors and the error rate of concentration under different wind directions.

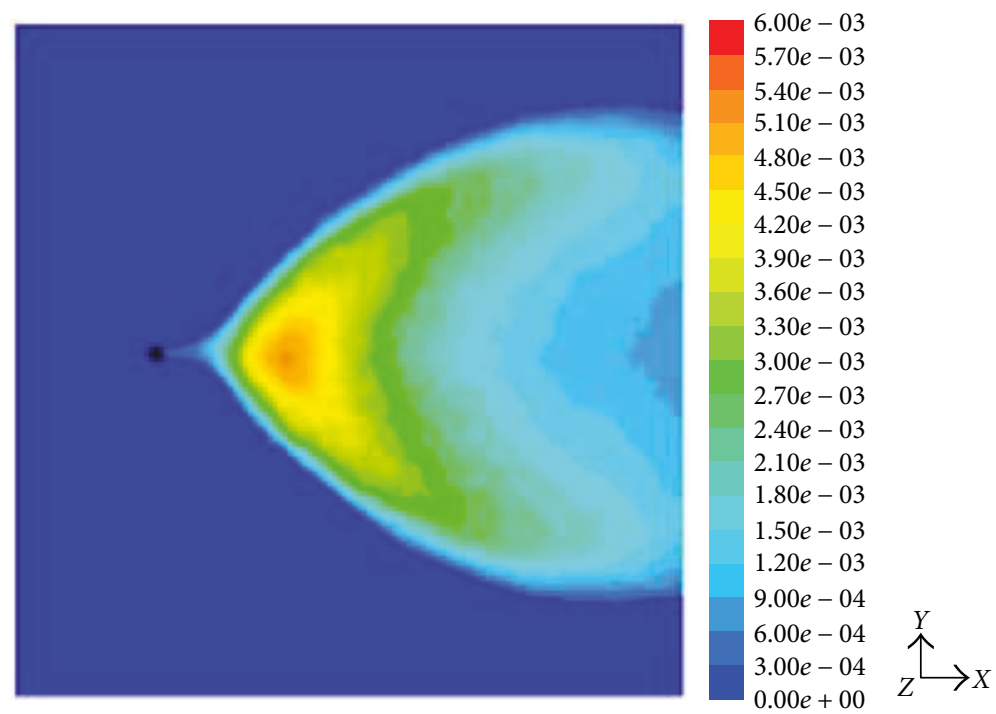

Figure 5: Diagram of gas diffusion situation.

According to Figures 5(b) and 5(d), the space between each row and each column is 5 meters, so the coordinates of the sensors are shown in Table 6.

According to Figures 5(c) and 5(e), the angle between the edge of the sector and horizontal direction is 30 degrees. The average space of the sector edge is 5 meters, where the sensors are deployed. So the coordinates of the sensors are shown in Table 7.

6.3. The Optimization Deployment Criterion. The optimization deployment criterion in this paper is that the more the number of alarm concentration points covered by the gas sensors, the better the deployment method, and meanwhile, the more balanced the coverage, the better the deployment method.

6.4. The Performance Analysis of Different Deployment Schemes. According to Tables 1, 5, 6, and 7 and the perceived radius $r=5 \mathrm{~m}$, we can obtain the coverage diagrams of different deployment methods, which are shown in Figures $7(a)-7(\mathrm{c})$.

As shown in Figure 7, the red spots represent the alarm concentration points, the black spots represent the 
TABLE 1: The coordinates and the concentration of the alarm concentration points.

\begin{tabular}{|c|c|c|c|}
\hline$X(\mathrm{~m})$ & $Y(\mathrm{~m})$ & $Z(\mathrm{~m})$ & $C\left(\mathrm{kmol} / \mathrm{m}^{3}\right)$ \\
\hline $4.000000000 E+001$ & $2.500000000 E+001$ & $6.000000238 E-001$ & $1.120437286 E-003$ \\
\hline $3.999999619 E+001$ & $3.000000000 E+001$ & $5.999999642 E-001$ & $1.264600200 E-003$ \\
\hline $4.000000000 E+001$ & $3.500000000 E+001$ & $6.000000238 E-001$ & $1.713989070 E-003$ \\
\hline $4.000000000 E+001$ & $4.000000000 E+001$ & $6.000000238 E-001$ & $1.921520452 E-003$ \\
\hline $4.500000000 E+001$ & $1.000000000 E+001$ & $6.000000834 E-001$ & $1.497431425 E-003$ \\
\hline $4.500000000 E+001$ & $1.500000000 E+001$ & $6.000000238 E-001$ & $1.519756159 E-003$ \\
\hline $4.500000000 E+001$ & $1.999999809 E+001$ & $6.000000238 E-001$ & $1.212779200 E-003$ \\
\hline $4.500000000 E+001$ & $2.499999809 E+001$ & $6.000000238 E-001$ & $9.467600612 E-004$ \\
\hline $4.500000000 E+001$ & $3.000000191 E+001$ & $6.000000834 E-001$ & $1.051027328 E-003$ \\
\hline $4.500000381 E+001$ & $3.500000000 E+001$ & $6.000000238 E-001$ & $1.397613552 E-003$ \\
\hline $4.500000000 E+001$ & $4.000000000 E+001$ & $5.999999642 E-001$ & $1.543087885 E-003$ \\
\hline $5.000000000 E+001$ & $1.000000000 E+001$ & $6.000000238 E-001$ & $1.001699828 E-003$ \\
\hline $5.000000000 E+001$ & $1.500000095 E+001$ & $6.000000238 E-001$ & $1.173341647 E-003$ \\
\hline $5.000000000 E+001$ & $2.000000000 E+001$ & $6.000000238 E-001$ & $9.443978197 E-004$ \\
\hline $5.000000000 E+001$ & $3.500000000 E+001$ & $5.999999642 E-001$ & $1.086011529 E-003$ \\
\hline $5.000000000 E+001$ & $4.000000000 E+001$ & $6.000000238 E-001$ & $1.094645238 E-003$ \\
\hline $1.499999905 E+001$ & $2.500000000 E+001$ & $6.000000238 E-001$ & $1.173134544 E-003$ \\
\hline $2.000000000 E+001$ & $2.000000000 E+001$ & $6.000000238 E-001$ & $2.732974943 E-003$ \\
\hline $2.000000000 E+001$ & $3.000000000 E+001$ & $6.000000238 E-001$ & $3.228770103 E-003$ \\
\hline $2.500000000 E+001$ & $1.500000000 E+001$ & $6.000000238 E-001$ & $1.646986115 E-003$ \\
\hline $2.499999809 E+001$ & $2.000000000 E+001$ & $6.000000238 E-001$ & $3.807903500 E-003$ \\
\hline $2.500000000 E+001$ & $2.499999809 E+001$ & $6.000000238 E-001$ & $3.564156126 E-003$ \\
\hline $2.500000000 E+001$ & $3.000000000 E+001$ & $6.000000238 E-001$ & $3.712610109 E-003$ \\
\hline $2.499999809 E+001$ & $3.500000000 E+001$ & $6.000000238 E-001$ & $2.364006825 E-003$ \\
\hline $3.000000000 E+001$ & $1.499999905 E+001$ & $6.000000238 E-001$ & $3.009808948 E-003$ \\
\hline $3.000000191 E+001$ & $2.000000000 E+001$ & $6.000000834 E-001$ & $2.742161509 E-003$ \\
\hline $3.000000000 E+001$ & $2.500000000 E+001$ & $6.000000238 E-001$ & $2.092821756 E-003$ \\
\hline $3.000000191 E+001$ & $3.000000000 E+001$ & $6.000000238 E-001$ & $2.553357044 E-003$ \\
\hline $3.000000000 E+001$ & $3.500000000 E+001$ & $6.000000238 E-001$ & $2.947377041 E-003$ \\
\hline $3.500000000 E+001$ & $1.000000000 E+001$ & $6.000000238 E-001$ & $1.484081615 E-003$ \\
\hline $3.500000000 E+001$ & $1.500000000 E+001$ & $6.000000238 E-001$ & $2.452870132 E-003$ \\
\hline $3.500000381 E+001$ & $2.000000191 E+001$ & $6.000001431 E-001$ & $1.859367127 E-003$ \\
\hline $3.500000000 E+001$ & $2.500000000 E+001$ & $6.000000834 E-001$ & $1.524172141 E-003$ \\
\hline $3.500000000 E+001$ & $3.000000000 E+001$ & $6.000000238 E-001$ & $1.704695402 E-003$ \\
\hline $3.500000000 E+001$ & $3.500000000 E+001$ & $6.000000238 E-001$ & $2.313728910 E-003$ \\
\hline $3.500000000 E+001$ & $4.000000000 E+001$ & $6.000000238 E-001$ & $1.883703284 E-003$ \\
\hline $4.000000000 E+001$ & $1.000000000 E+001$ & $6.000000238 E-001$ & $1.793242875 E-003$ \\
\hline $4.000000000 E+001$ & $1.500000000 E+001$ & $6.000000238 E-001$ & $1.869315282 E-003$ \\
\hline $4.000000000 E+001$ & $1.999999809 E+001$ & $6.000000238 E-001$ & $1.410081866 E-003$ \\
\hline
\end{tabular}

TABLE 2: The values of $A$ and $B$ calculated according to the number of gas sensors.

\begin{tabular}{lccccc}
\hline Number of gas sensors & 5 & 8 & 11 & 14 & 17 \\
\hline$A$ & 0.3200 & 0.2188 & 0.3306 & 0.2653 & 0.1107 \\
$B$ & 0.7692 & 0.6667 & 0.4872 & 0.3590 & 0.5385 \\
\hline
\end{tabular}

TABLE 3: The standardized values of $A$ and $B$ calculated according to the number of gas sensors.

\begin{tabular}{lccccc}
\hline Number of gas sensors & 5 & 8 & 11 & 14 & 17 \\
\hline$A$ & 0.2938 & 0.2471 & 0.4043 & 0.4250 & 0.1705 \\
$B$ & 0.7062 & 0.7529 & 0.5957 & 0.5750 & 0.8295 \\
\hline
\end{tabular}


TABle 4: The weight values of the $A$ and $B$.

\begin{tabular}{lcc}
\hline & $\alpha$ & $\beta$ \\
\hline Weight value & 0.4886 & 0.5114 \\
\hline
\end{tabular}

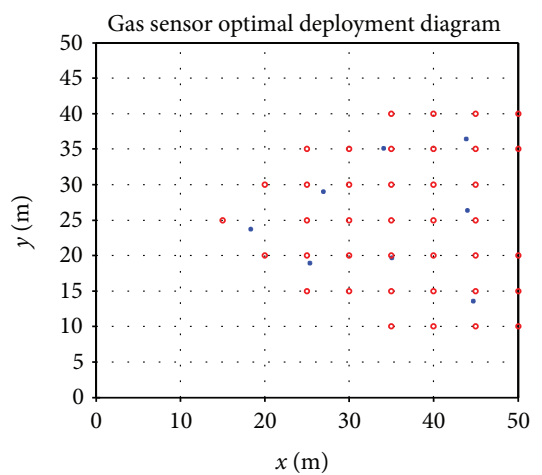

Alarm concentration point

* Sensor

(a)

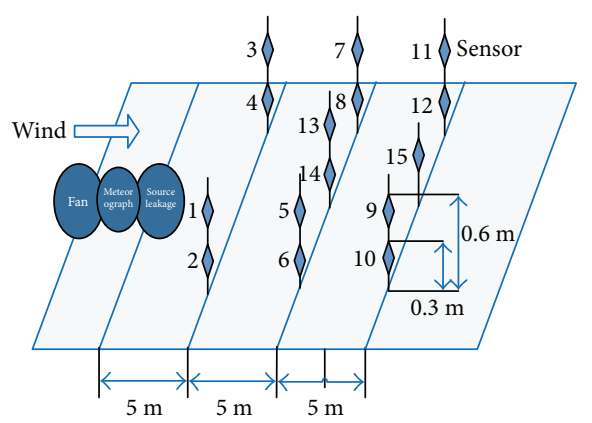

(b)

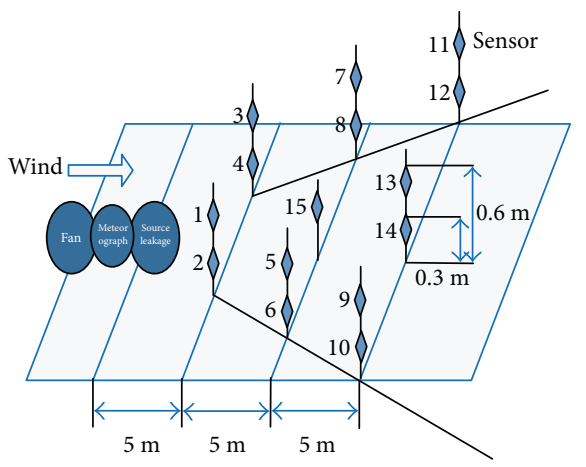

(c)

FIGURE 6: (a) The gas sensor deployment diagram based on the PSO algorithm. (b) The rectangular gas sensor deployment diagram according to the standard. (c) The sector gas sensor deployment diagram according to the standard.

TABLE 5: The coordinates of gas sensors in the optimal deployment scheme based on the PSO algorithm.

\begin{tabular}{ccccccccc}
\hline & S1 & S2 & S3 & S4 & S5 & S6 & S7 & S8 \\
\hline$X(\mathrm{~m})$ & 43.84 & 44.67 & 26.95 & 34.05 & 44.03 & 25.34 & 35.04 & 18.34 \\
$Y(\mathrm{~m})$ & 36.45 & 13.57 & 29.01 & 35.10 & 26.37 & 18.96 & 19.68 & 23.74 \\
$Z(\mathrm{~m})$ & 0.60 & 0.60 & 0.60 & 0.60 & 0.60 & 0.60 & 0.60 & 0.60 \\
\hline
\end{tabular}

TABLE 6: The coordinates of the sensors and the corresponding concentration according to the standard rectangular deployment method.

\begin{tabular}{lcccccccc}
\hline & $\mathrm{R} 1$ & $\mathrm{R} 2$ & $\mathrm{R} 3$ & $\mathrm{R} 4$ & $\mathrm{R} 5$ & $\mathrm{R} 6$ & $\mathrm{R} 7$ & $\mathrm{R} 8$ \\
\hline$X(\mathrm{~m})$ & 15 & 15 & 35 & 35 & 35 & 40 & 40 & 40 \\
$Y(\mathrm{~m})$ & 20 & 30 & 20 & 25 & 30 & 20 & 25 & 30 \\
$Z(\mathrm{~m})$ & 0.60 & 0.60 & 0.60 & 0.60 & 0.60 & 0.60 & 0.60 & 0.60 \\
\hline
\end{tabular}

TABLE 7: The coordinates of the sensors and the corresponding concentration according to the standard sector deployment method.

\begin{tabular}{ccccccccc}
\hline & D1 & D2 & D3 & D4 & D5 & D6 & D7 & D8 \\
\hline$X(\mathrm{~m})$ & 15 & 15 & 20 & 20 & 25 & 25 & 30 & 30 \\
$Y(\mathrm{~m})$ & 22.11 & 27.88 & 19.23 & 30.77 & 16.34 & 33.66 & 20 & 30 \\
$Z(\mathrm{~m})$ & 0.60 & 0.60 & 0.60 & 0.60 & 0.60 & 0.60 & 0.60 & 0.60 \\
\hline
\end{tabular}

gas sensors deployed, and the black circles represent the perceived radius.

According to (9) and (10), we can calculate the number of the alarm concentration points covered by the gas sensors in different deployment methods and the coverage balance degree, which are shown in Table 8.

According to (10), $A=\sum_{j=1}^{N} \sqrt{\left(c\left(s_{j}\right)-E(s)\right)^{2}} / N$, we can know that the smaller $A$ is, the better the coverage balance degree is, as shown in Table 8. The number of the alarm concentration points covered by the gas sensors in the optimization deployment method based on the PSO algorithm is 28 , and it is larger than the number of the alarm concentration points covered by the gas sensors in other two deployment methods. And the coverage balance degree $A$ is 0.6562 , and it is less than the values of $A$ in other two deployment methods. Thus, the deployment method we proposed is the best; therefore, we improve the monitor efficiency of the gas sensor networks.

\section{Conclusions}

In the chemical industrial park, the gas sensors installed in accordance with the traditional standards may not detect gas leakage efficiently due to the nondeterministic deployment. And the problems of coverage hole and repeated coverage will happen. Aimed at these problems, this paper proposes a deterministic deployment method taking the wind speed and direction into consideration based on the gas diffusion model, then we research the influence of the wind speed and direction on the error rate of concentration in the deployment method. The results are as follows: the variation of the wind speed is larger, the error rate of concentration is larger, and the variation of wind direction is larger, and the error rate of concentration is smaller. After that, under the main wind speed and direction somewhere condition, we firstly put forward a new deterministic deployment method of gas sensors, use the theory of CFD to simulate the gas diffusion situation, and propose a new optimization deployment criterion, namely, the more alarm concentration points are covered by gas sensors, the more balance the 


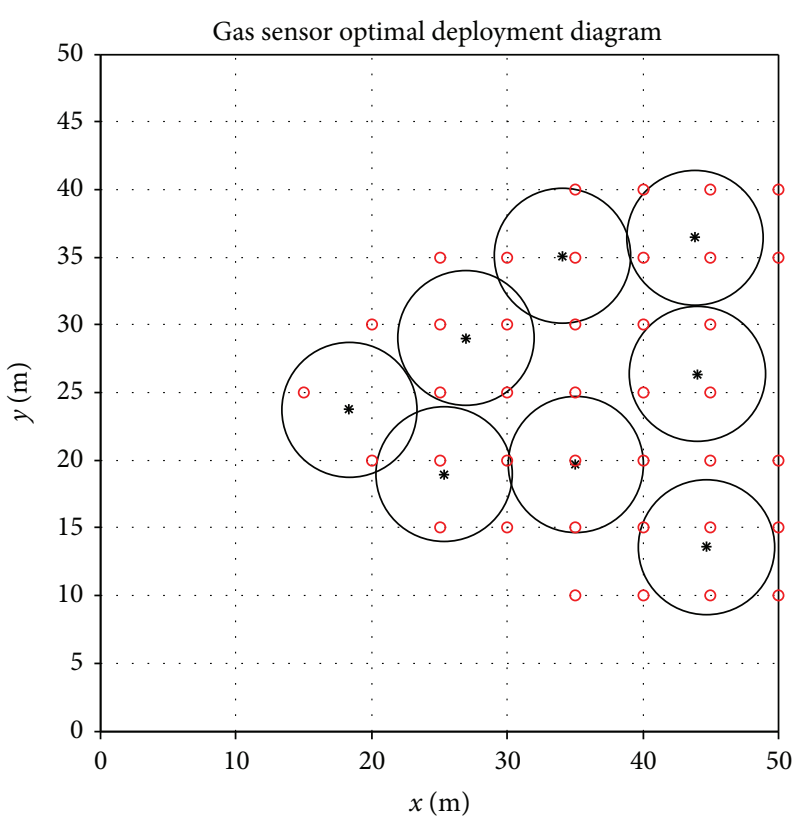

$\bigcirc$ Alarm concentration point

* Sensor

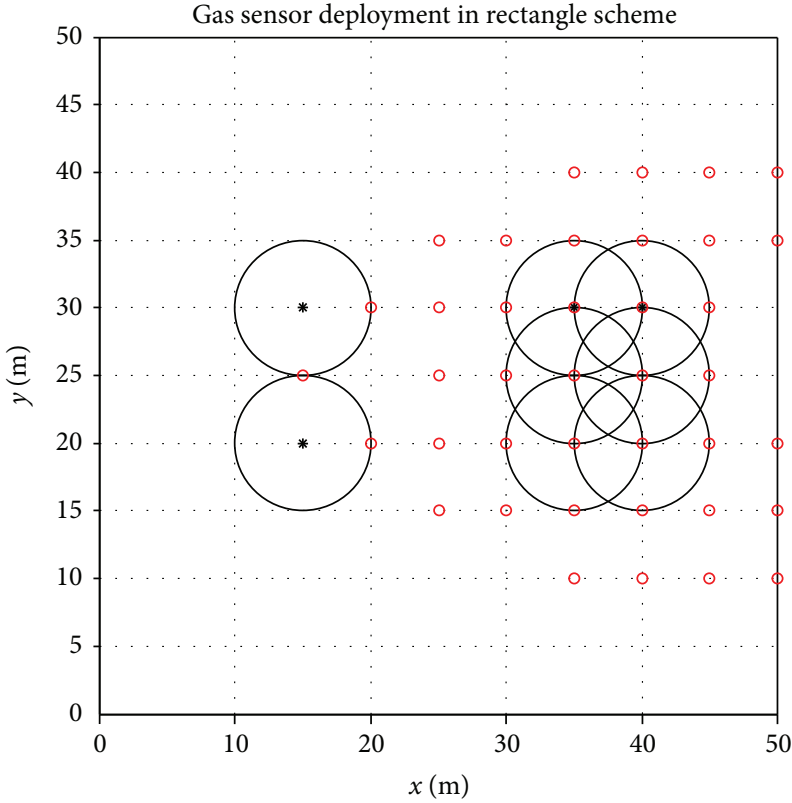

O Alarm concentration point

* Sensor

(a)

(b)

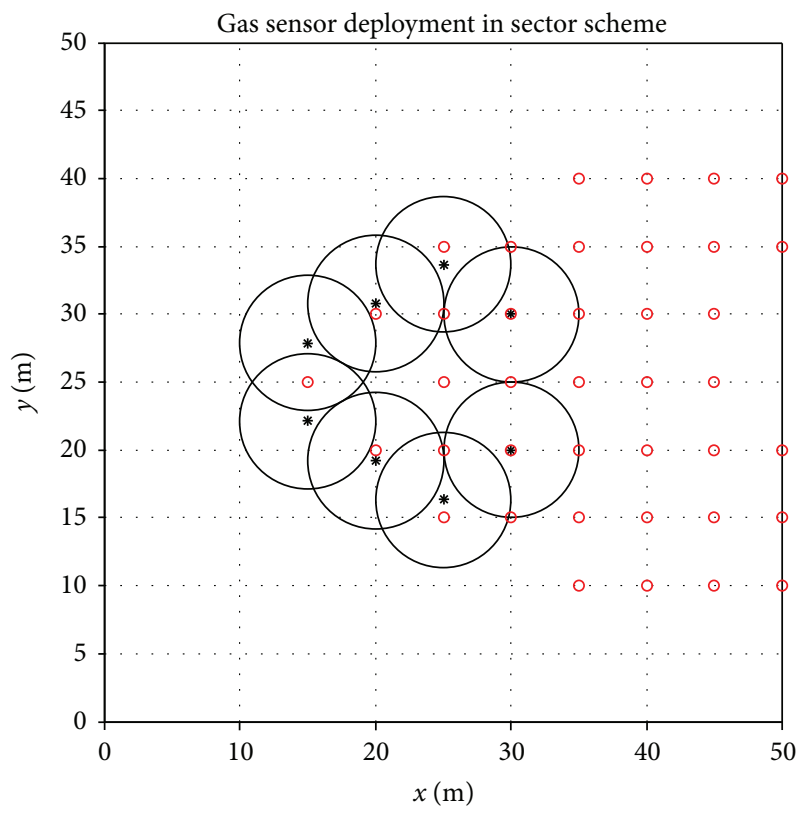

O Alarm concentration point

* Sensor

(c)

Figure 7: (a) The coverage diagram of the optimization deployment method based on the PSO algorithm. (b) The coverage diagram of rectangular gas sensor deployment method according to the standard. (c) The coverage diagram of sector gas sensor deployment method according to the standard.

coverage is, and the deployment method is more optimal, and then a new objective function is built based on the criteria. Secondly, we obtain the weight values of the function using entropy estimation method. Finally, we deploy the gas sensors determinately using the particle swarm optimization (PSO) algorithm. The simulation results show that the coverage degree of the proposed deployment method is $71.79 \%$, the coverage balance degree is 0.625 , the coverage degree of the rectangular deployment method is $48.72 \%$, the coverage balance degree is 0.8125 , the coverage degree of the sector deployment method is $35.80 \%$, and the coverage balance degree is 1.2813 . We know that the less the value of 
TABLE 8: The number of the alarm concentration points covered by gas sensors and the coverage balance degree.

\begin{tabular}{lccc}
\hline Methods & $\begin{array}{c}\text { Optimization } \\
\text { method }\end{array}$ & $\begin{array}{c}\text { Rectangular } \\
\text { deployment } \\
\text { method }\end{array}$ & $\begin{array}{c}\text { Sector } \\
\text { deployment } \\
\text { method }\end{array}$ \\
\hline $\begin{array}{l}\text { The number of the } \\
\text { covered alarm } \\
\text { concentration points }\end{array}$ & $\mathbf{2 8}$ & 19 & 14 \\
$\begin{array}{l}\text { Coverage degree } \\
\begin{array}{l}\text { Coverage balance } \\
\text { degree }(A)\end{array}\end{array}$ & $\mathbf{7 1 . 7 9 \%}$ & $48.72 \%$ & $35.89 \%$ \\
\hline
\end{tabular}

coverage balance degree is, the better the performance of the deployment method is. Therefore, the deployment method we proposed in this paper can improve the coverage performance and meanwhile improve the coverage balance degree. In other words, it can enhance the monitor efficiency. And the deployment method we proposed is of great relevance and guidance to the current deployment methods.

\section{Conflicts of Interest}

The authors declare no conflicts of interest.

\section{Acknowledgments}

This project was supported by "the collaborative project support for technology and education" of the Chinese Academy of Sciences (Y426473231).

\section{References}

[1] Health and Safety Commission Annual Report and the Health and Safety Commission/Executive Accounts 04, London, The Stationery Office, London, UK, 2003.

[2] Y. Guo, F. Kong, D. Zhu, A. Ş. Tosun, and Q. Deng, "Sensor placement for lifetime maximization in monitoring oil pipelines," in Proceedings of the 1st ACM/IEEE International Conference on Cyber-Physical Systems, pp. 61-68, New York, NY, USA, 2010.

[3] T. T. Lai, W. J. Chen, K. H. Li, P. Huang, and H.-H. Chu, "TriopusNet: automating wireless sensor network deployment and replacement in pipeline monitoring," in 2012 ACM/IEEE, 11th International Conference on Information Processing in Sensor Networks (IPSN), pp. 61-71, Beijing, China, 2012.

[4] M. T. Kouakou, K. Yasumoto, S. Yamamoto, and M. Ito, "Cost-efficient sensor deployment in indoor space with obstacles," in 2012 IEEE International Symposium on a World of Wireless, Mobile and Multimedia Networks (WoWMoM), pp. 1-9, San Francisco, CA, USA, 2012.

[5] M. T. Kouakou, S. Yamamoto, K. Yasumoto, and M. Ito, "Deployment planning tool for indoor 3D-WSNs," in Proceedings of the 12th ACM international conference adjunct papers on ubiquitous computing-Ubicomp '10, pp. 369-370, Copenhagen, Denmark, September, 2010.

[6] M. Huchard, V. Paquier, A. Loeillet, V. Marangozov, and J.-M. Nicolai, "Indoor deployment of a wireless sensor network for inventory and localization of mobile assets," in 2012 IEEE International Conference on RFID-Technologies and Applications (RFID-TA), pp. 369-372, Nice, France, 2012.
[7] J. Heidemann, M. Stojanovic, and M. Zorzi, "Underwater sensor networks: applications, advances and challenges," Philosophical Transactions of the Royal Society A, vol. 370, no. 1958, pp. 158-175, 2012.

[8] R. Huang, W. Z. Song, M. Xu, N. Peterson, B. Shirazi, and R. LaHusen, "Real-world sensor network for long-term volcano monitoring: design and findings," IEEE Transactions on Parallel and Distributed Systems, vol. 23, no. 2, pp. 321-329, 2012.

[9] Y. Wang, W. Fu, and D. P. Agrawal, "Gaussian versus uniform distribution for intrusion detection in wireless sensor networks," IEEE Transactions on Parallel and Distributed Systems, vol. 24, no. 2, pp. 342-355, 2013.

[10] X. Li, L. Ci, M. Yang, C. Tian, and X. Li, "Deploying threedimensional mobile sensor networks based on virtual forces algorithm," in China Conference on Wireless Sensor Networks, pp. 204-216, Huangshan, China, October, 2012.

[11] S. De Friend, M. Dejmek, L. Porter, B. Deshotels, and B. Natvig, "A risk-based approach to flammable gas detector spacing," Journal of Hazardous Materials, vol. 159, no. 1, pp. 142-151, 2008.

[12] S. W. Legg, A. J. Benavides-Serrano, J. D. Siirola et al., "A stochastic programming approach for gas detector placement using CFD-based dispersion simulations," Computers \& Chemical Engineering, vol. 47, pp. 194-201, 2012.

[13] R. Li, X. Liu, W. Xie, and N. Huang, "Deployment-based lifetime optimization model for homogeneous wireless sensor network under retransmission," Sensors, vol. 14, no. 12, pp. 23697-23724, 2014

[14] L. Liu, O. Masfary, and N. Antonopoulos, "Energy performance assessment of virtualization technologies using small environmental monitoring sensors," Sensors, vol. 12, no. 5, pp. 6610-6628, 2012.

[15] P. Jiang, X. Wang, and L. Jiang, "Node deployment algorithm based on connected tree for underwater sensor networks," Sensors, vol. 15, no. 7, pp. 16763-16785, 2015.

[16] Y. Jiang, Z. He, Y. Li, Z. Xu, and J. Wei, "Weighted global artificial bee colony algorithm makes gas sensor deployment efficient," Sensors, vol. 16, no. 6, p. 888, 2016.

[17] V. Vassiliou and C. Sergiou, "Performance study of node placement for congestion control in wireless sensor networks," in 2009 3rd International Conference on New Technologies, Mobility and Security, pp. 1-8, Cairo, Egypt, December, 2009.

[18] R. Qin, Q. Sun, X. You, and X. Yuan, “A key management scheme for manually deployed wireless sensor networks based on dual directional hash chains," in 2012 Second International Conference on Intelligent System Design and Engineering Application, pp. 752-755, Sanya, Haian, China, January 2012.

[19] C. Liu and G. Cao, "Spatial-temporal coverage optimization in wireless sensor networks," IEEE Transactions on Mobile Computing, vol. 10, no. 4, pp. 465-478, 2011.

[20] C. Ozturk, D. Karaboga, and B. Gorkemli, "Probabilistic dynamic deployment of wireless sensor networks by artificial bee colony algorithm," Sensors, vol. 11, no. 6, pp. 6056-6065, 2011.

[21] H. M. Ammari and S. Das, "A study of k-coverage and measures of connectivity in 3D wireless sensor networks," IEEE Transactions on Computers, vol. 59, no. 2, pp. 243-257, 2010.

[22] H. González-Baños, "A randomized art-gallery algorithm for sensor placement," in Proceedings of the seventeenth annual 
symposium on Computational geometry-SCG '01, pp. 232-240, Medford, MA, USA, June, 2001.

[23] J. M. Gil and Y. H. Han, "A target coverage scheduling scheme based on genetic algorithms in directional sensor networks," Sensors, vol. 11, no. 2, pp. 1888-1906, 2011.

[24] J. O'rourke, Art Gallery Theorems and Algorithms, Oxford University Press, New York, NY, USA, 1987.

[25] H. Mostafaei and M. R. Meybodi, "Maximizing lifetime of target coverage in wireless sensor networks using learning automata," Wireless Personal Communications, vol. 71, no. 2, pp. 1461-1477, 2013.

[26] Z. Lu and W. W. Li, "Approximation algorithms for maximum target coverage in directional sensor networks," in 2014 IEEE 11th International Conference on Networking, Sensing and Control (ICNSC), pp. 155-160, Miami, FL, USA, April 2014.

[27] H. N. Djidjev, "Approximation algorithms for computing minimum exposure paths in a sensor field," ACM Transactions on Sensor Networks, vol. 7, no. 3, pp. 1-25, 2010.

[28] C. Lee, D. Shin, S. W. Bae, and S. Choi, "Best and worst-case coverage problems for arbitrary paths in wireless sensor networks," Ad Hoc Networks, vol. 11, no. 6, pp. 1699-1714, 2013.

[29] G. S. Kasbekar, Y. Bejerano, and S. Sarkar, "Lifetime and coverage guarantees through distributed coordinate-free sensor activation," IEEE/ACM Transactions on Networking, vol. 19, no. 2, pp. 470-483, 2011.

[30] X. Li, G. Fletcher, A. Nayak, and I. Stojmenovic, "Placing sensors for area coverage in a complex environment by a team of robots," ACM Transactions on Sensor Networks, vol. 11, no. 1, pp. 1-22, 2014.

[31] Y. E. Osais, M. St-Hilaire, and F. R. Yu, "Directional sensor placement with optimal sensing range, field of view and orientation," Mobile Networks and Applications, vol. 15, no. 2, pp. 216-225, 2010.

[32] J. Kennedy, R. Eberhat, and Y. Shi, Swarm Intelligence, Morgan Kaufmann Academic Press, Burlington, MA, USA, 2001.

[33] J. J. Liang, A. K. Qin, P. N. Suganthan, and S. Baskar, "Comprehensive learning particle swarm optimizer for global optimization of multimodal functions," IEEE Transactions on Evolutionary Computation, vol. 10, no. 3, pp. 281-295, 2006.

[34] Y. Shi and R. Eberhart, "A modified particle swarm optimizer," in 1998 IEEE International Conference on Evolutionary Computation Proceedings. IEEE World Congress on Computational Intelligence, Anchorage, AK, USA, 1998.

[35] Y. Shi and R. Eberhart, "Empirical study of particle swarm optimization," in Proceedings of the 1999 Congress on Evolutionary Computation-CEC99, Washington, DC, USA, 1999.

[36] Y. Shi and R. Eberhart, "Fuzzy adaptive particle swarm optimization," in Proceedings of the 2001 Congress on Evolutionary Computation, Seoul, South Korea, 2001.

[37] J. Matthes, L. Groll, and H. B. Keller, "Source localization based on pointwise concentration measurements," Sensors and Actuators A: Physical, vol. 115, no. 1, pp. 32-37, 2004.

[38] J. Matthes, L. Groll, and H. B. Keller, "Source localization by spatially distributed electronic noses for advection and diffusion," IEEE Transactions on Signal Processing, vol. 53, no. 5, pp. 1711-1719, 2005.

[39] H. Du, N. Xia, and R. Zheng, "Particle swarm inspired underwater sensor self-deployment," Sensors, vol. 14, no. 8, pp. 15262-15281, 2014. 


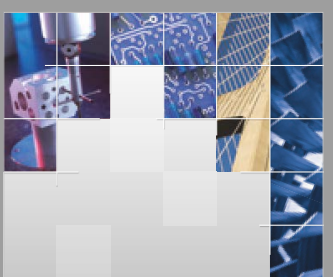

\section{Enfincering}
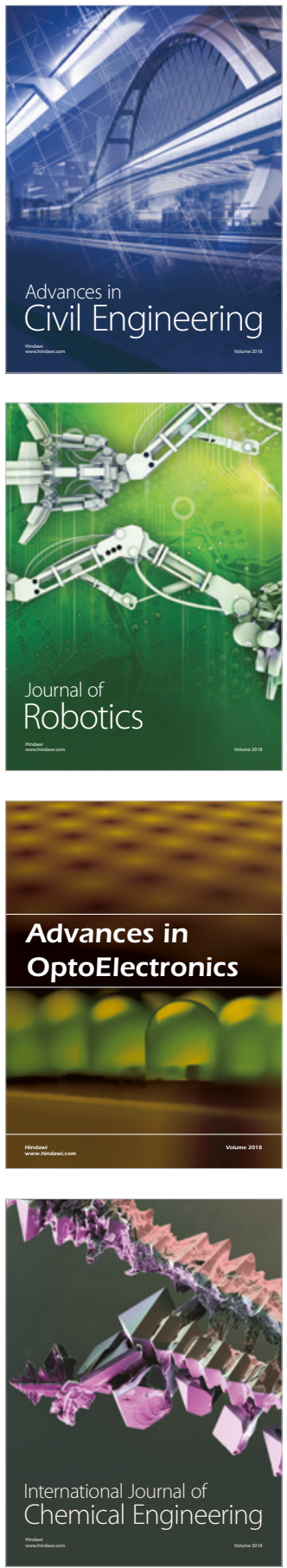

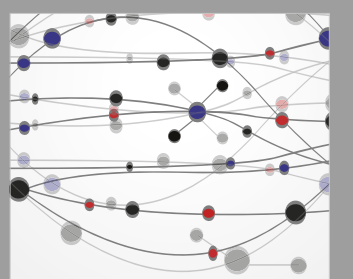

\section{Rotating \\ Machinery}

The Scientific World Journal

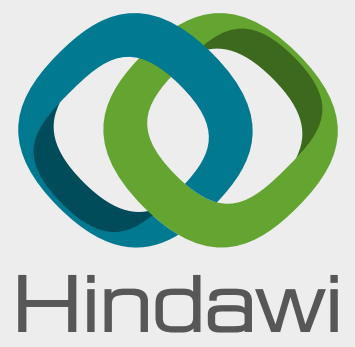

Submit your manuscripts at

www.hindawi.com
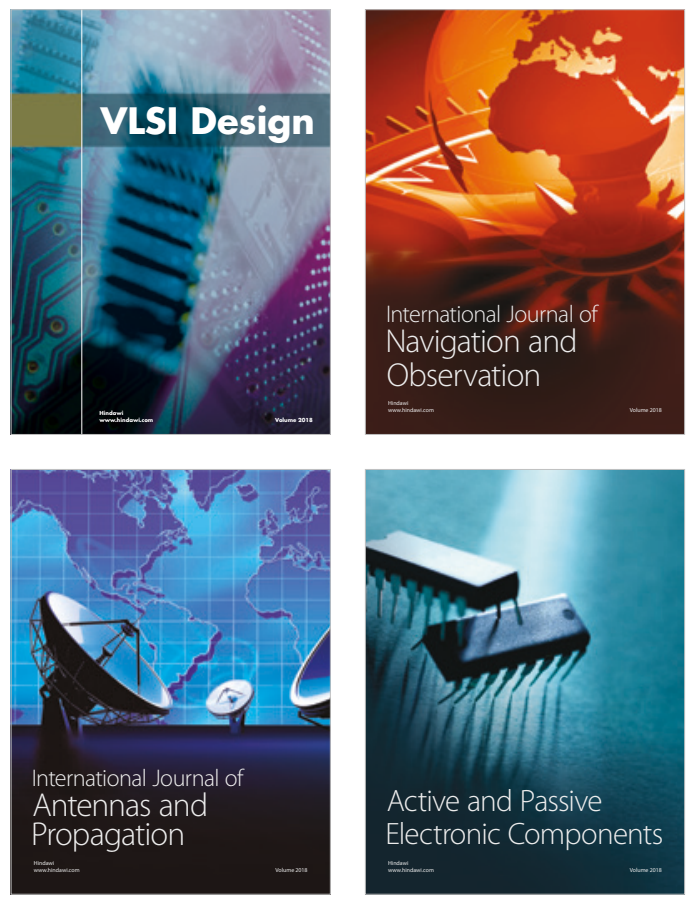
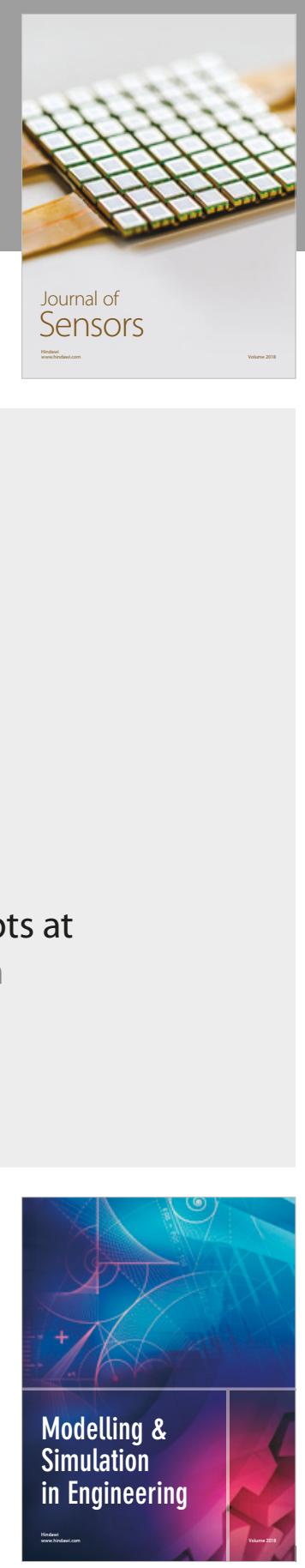

\section{Advances \\ Multimedia}
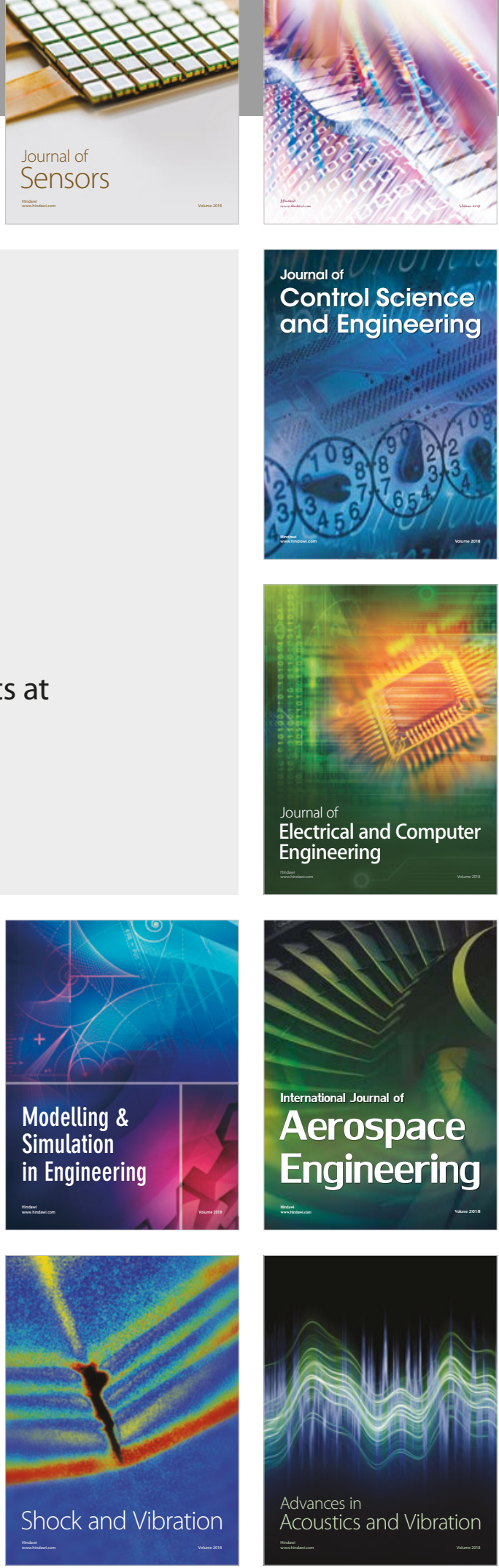